\title{
Corrigendum: Oncogenic NRAS signaling differentially regulates survival and proliferation in melanoma
}

Lawrence N Kwong, James C Costello, Huiyun Liu, Shan Jiang, Timothy L Helms, Aliete E Langsdorf, David Jakubosky, Giannicola Genovese, Florian L Muller, Joseph H Jeong, Ryan P Bender, Gerald C Chu, Keith T Flaherty, Jennifer A Wargo, James J Collins \& Lynda Chin

Nat. Med. 18, 1503-1510 (2012); published online 16 September 2012; corrected after print 20 November 2012

In the version of this article initially published, the formula to calculate the mutual information matrix, which appears in the last page of the Online Methods, was missing a log multiplier. The correct formula is below, and the error has been corrected in the HTML and PDF versions of the article.

$$
I(X ; Y)=\sum_{i, j} P\left(x_{i}, y_{j}\right) \times \log \left(\frac{P\left(x_{i}, y_{j}\right)}{P\left(x_{i}\right) \times P\left(y_{j}\right)}\right)
$$

\section{Corrigendum: Sarcolipin is a newly identified regulator of muscle-based thermogenesis in mammals}

\footnotetext{
Naresh C Bal, Santosh K Maurya, Danesh H Sopariwala, Sanjaya K Sahoo, Subash C Gupta, Sana A Shaikh, Meghna Pant, Leslie A Rowland, Sanjeewa A Goonasekera, Jeffery D Molkentin \& Muthu Periasamy Nat. Med. 18, 1575-1579 (2012); published online 9 September 2012; corrected after print 6 December 2012
}

The authors would like to add two co-authors, A. Russell Tupling and Eric Bombardier, to the study. The author list, Acknowledgments and Author Contributions have been corrected in the HTML and PDF versions of this article. 